INPLASY

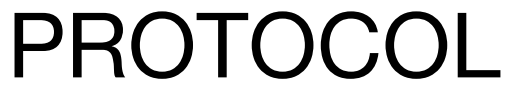

To cite: Zhao et al. Efficacy and Safety of Fingolimod in Stroke: A Systemic Review and Meta-analysis. Inplasy protocol 202140130. doi:

10.37766/inplasy2021.4.0130

Received: 25 April 2021

Published: 25 April 2021

Corresponding author:

Kai Zhao

15544980059@163.com

Author Affiliation:

Qinghai University

Support: Project of Qinghai Province.

Review Stage at time of this submission: Data analysis.

Conflicts of interest:

None declared.

\section{Efficacy and Safety of Fingolimod in Stroke: A Systemic Review and Meta-analysis}

Zhao, K; Guo, Y2; Zhang, Q3; Yang, M4.

Review question / Objective: We tried to make a systemic review and meta-analysis of recent random control trials to confirm our hypothesis that clinical application of fingolimod could promote reduction of infarction lesion or hematoma and improve neurological prognosis in stroke patients via reducing lymphocyte infiltration effectively and safely.

Condition being studied: It had been a conclusion from the meta-analysis of animal experiments that fingolimod could treat animal models of stroke via reducing lymphocyte infiltration effectively.

Information sources: Three open electronic database of PubMed, Embase and Cochrane were used to make the accomplishment of literature search comprehensively.

INPLASY registration number: This protocol was registered with the International Platform of Registered Systematic Review and Meta-Analysis Protocols (INPLASY) on 25 April 2021 and was last updated on 25 April 2021 (registration number INPLASY202140130).

\section{INTRODUCTION}

Review question / Objective: We tried to make a systemic review and meta-analysis of recent random control trials to confirm our hypothesis that clinical application of fingolimod could promote reduction of infarction lesion or hematoma and improve neurological prognosis in stroke patients via reducing lymphocyte infiltration effectively and safely.

Rationale: Stroke, which mainly consists of acute ischemic stroke(AIS) with cerebral infarction and hemorrhagic stroke with intracerebral hematoma(ICH), is a common disease with high mortality and disability in neurology, especially, the incidence of 
stroke was increased significantly during the coronavirus-2019 pneumonia pandemic, which aggravated financial burden in the world. Severe stroke patients whose condition took a sharp turn for the worse must be survived via surgical intervention, which would be only the best way to save lives. Thus, neurologists have turned their attention to timely intervention to the early stage of stroke through medical treatment for reducing the risk of condition deteriorated and promoting the recovery of nervous system function. Fingolimod is a new medicine to treat leukoencephalopathy. After oral medication, Fingolimod could be metabolized to fingolimod-phosphate as the active metabolite via sphingosine kinase. Sphingosine-1-phosphate receptor 1, 3, 4 and 5 could be bound to fingolimodphosphate due to its high affinity. Sphingosine-1-phosphate and its receptors could regulate lymphocyte migration via upstream cell signaling pathways. Thus, fingolimod-phosphate could block the ability of entrance or exit to lymph nodes in lymphocytes, which could reduce the number of lymphocytes in peripheral blood. Although fingolimod could perform therapy of multiple sclerosis with unknown mechanism, its role of immunomodulator might involve the reduction of lymphocytes migrated to central nervous system. Brain tissue injury in stroke patients involved inflammation around infarction lesion or hematoma, which was an important reason to deteriorate condition early and result to poor prognosis. It had been a conclusion from the meta-analysis of animal experiments that fingolimod could treat animal models of stroke via reducing lymphocyte infiltration effectively. Yet the efficacy and safety of fingolimod applied to stroke patients has not been found in evidence-based medicine. We tried to make a systemic review and meta-analysis of recent random control trials to confirm our hypothesis that clinical application of fingolimod could promote reduction of infarction lesion or hematoma and improve neurological prognosis in AIS or $\mathrm{ICH}$ patients via reducing lymphocyte infiltration effectively and safely.
Condition being studied: It had been a conclusion from the meta-analysis of animal experiments that fingolimod could treat animal models of stroke via reducing lymphocyte infiltration effectively.

\section{METHODS}

Search strategy: Here was the strategy of literature search on the Pubmed: ("Fingolimod Hydrochloride"[Mesh]) OR (((((2-Amino-2-(2-(4-octylphenyl)ethyl)-1,3propanediol hydrochloride[Title/Abstract]) OR (FTY?720[Title/Abstract])) OR (Gilenya[Title/Abstract])) OR (Gilenia[Title/ Abstract])) OR (Fingolimod[Title/Abstract])) AND (( "Stroke "[M esh]) OR

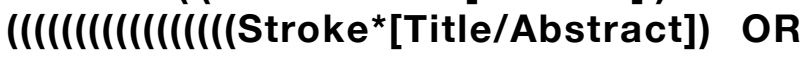
(Cerebrovascular Accident*[Title/ Abstract])) OR (CVA* [Title/Abstract])) OR (Cerebrovascular Apoplexy[Title/Abstract])) OR (Apoplexy, Cerebrovascular[Title) Abstract])) OR (Vascular Accident, Brain[Title/Abstract])) OR (Brain Vascular Accident*[Title/Abstract])) OR (Vascular Accidents, Brain[Title/Abstract])) OR (Cerebrovascular Stroke*[Title/Abstract])) OR (Stroke*, Cerebrovascular[Title/ Abstract])) OR (Apoplexy[Title/Abstract])) OR (Cerebral Stroke*[Title/Abstract])) OR (Stroke*, Cerebral[Title/Abstract])) OR (Stroke*, Acute[Title/Abstract])) OR (Acute Stroke* [Title/Abstract])) OR (Cerebrovascular Accident ${ }^{\star}$, Acute[Title/ Abstract])) OR (Acute Cerebrovascular Accident ${ }^{\star}[$ Title/Abstract $\left.\left.\left.]\right)\right)\right)$.

Participant or population: Patients suffered from stroke which contains AIS or ICH.

Intervention: Application of fingolimod was the clinical intervention.

Comparator: No application of fingolimod was the clinical intervention.

Study designs to be included: Random control trials

Eligibility criteria: (1)Duplication; (2)Reviews, comments, letters, case reports, protocols of clinic trials and conference papers; (3)Animal experiments; 
(4)All fields of the articles were not in accord with the subject.

Information sources: Three open electronic database of PubMed, Embase and Cochrane were used to make the accomplishment of literature search comprehensively.

Main outcome(s): Outcomes involved assessment of immunology, lesion volume neurological function and adverse events.

Additional outcome(s): In addition, some confounders, which might result to errors, were concluded: characters of participation, period of treatment and other factors.

Quality assessment / Risk of bias analysis: The quality assessment of literature included was performed via the Cochrane Collaboration's Tool of Assessing Risk of Bias by the software Review Manager 5.3 before data extraction.

Strategy of data synthesis: It was significant to actualize accurate, objective and scientific assessment of the curative effect and the safety of fingolimod applied to stroke patients. Our team had organized a conference to decide the method of assessment with reference of many document literature. In consideration of the pharmacological action of fingolimod, absolute immunological cell counts in blood might be the most intuitive quota. Especially, T-lymphocytes with cluster of differentiation 8 expression(CD8+ T cells, $\times 106 / \mathrm{ml}$ ) played the key role of damage in the nervous system due to its effect of cell killer, which resulted in inflammatory edema. The edema around infarction or hematoma could result to serious neurologic impairment in stroke patients. According to that, rapid reduction of absolute infarction or hematoma volume might be the key of treatment in stroke patients. Absolute lesion volume was defined to infarction volume in AIS patients or hematoma in $\mathrm{ICH}$ patients. National Institute of Health stroke scale(NIHSS) is the specialized assessment to measure recovery of nerve function. Modified
Barthel index $(\mathrm{mBI})$ is one of the earliest and the most commonly used methods of assess activity of daily living(ADL) in rehabilitation medicine with high validity and reliability in assessment of stroke. Though risk ratio(RR) is often used as an effect quantity in cohort studies, it might be significant to assess the probability of adverse events like the number of patients need to be harmed(NNH) in clinical trials. Thus, we decided that the data extraction of efficacy consisted of absolute CD8+ T cells counts, absolute lesion volume(infarction or hematoma), NIHSS, $\mathrm{mBI}$ and the data extraction of safety was RR. Referred to treatment periods of studies, we decided to choose the data in time points near baseline, which might reflect to condition of stroke in early treatment. In addition, some confounders, which might result to errors, were concluded: characters of participation, period of treatment and other factors. If the absolute data could not be acquired, other data were also be extracted such as the difference between data of baseline and data of other time point, relative data of quotas above, et al.

Subgroup analysis: In addition, some confounders, which might result to errors, were concluded: characters of participation, period of treatment and other factors.

Sensitivity analysis: Sensitivity analysis, the overall SMD or pooled effect of the rest studies were retrieved after the study with the highest quality was omitted or the fixed effects model was switched to random effects model, was the way to checkout the stability of overall results.

Language: Language of articles, regions were not restricted.

Country(ies) involved: China.

Other relevant information: None.

Keywords: Acute Ischemic Stroke, Intracerebral Hematoma, Fingolimod, Meta-analysis, Treatment. 
Dissemination plans: Published on Neurosurgical Review.

Contributions of each author:

Author 1 - Kai Zhao - Mingfei Yang and Kai Zhao conceived the study. Kai Zhao and Yu Guo screened studies and extracted the data independently. Kai Zhao and Yu Guo wrote the first draft of the manuscript.

Email: 15544980059@163.com

Author 2 - Yu Guo - Kai Zhao and Yu Guo screened studies and extracted the data independently. Kai Zhao and Yu Guo wrote the first draft of the manuscript.

Email: 15751445670@163.com

Author 3 - Qiang Zhang - Qiang Zhang and Yu Guo analysed and interpreted the data.

Email: zhangqiang691212@163.com

Author 4 - Mingfei Yang - Mingfei Yang and Kai Zhao conceived the study. Mingfei Yang proofread the manuscript before submission.

Email: iloveyoucmu@163.com 